\title{
A Truncated Singular Value Decomposition Enhanced Nested Complex Source Beam Method
}

\author{
Lianning Song and Zaiping Nie \\ Department of Microwave Engineering, University of Electronic Science and Technology of China, Chengdu, Sichuan 610054, China \\ Correspondence should be addressed to Lianning Song; lianningsong@foxmail.com
}

Received 15 May 2017; Accepted 8 June 2017; Published 1 August 2017

Academic Editor: Yumao Wu

Copyright (c) 2017 Lianning Song and Zaiping Nie. This is an open access article distributed under the Creative Commons Attribution License, which permits unrestricted use, distribution, and reproduction in any medium, provided the original work is properly cited.

\begin{abstract}
This work presents a novel matrix compression algorithm to improve the computational efficiency of the nested complex source beam (NCSB) method. The algorithm is based on the application of the truncated singular value decomposition (TSVD) to the multilevel aggregation, translation, and disaggregation operations in NCSB. In our implementation, the aggregation/disaggregation matrices are solved by the truncated far-field matching, which is based on the directional far-field radiation property of the complex source beams (CSBs). Furthermore, the translation matrices are obtained according to the beam width of CSBs. Due to the high directivity of the radiation patterns of CSBs, all the far-field related interaction matrices are low-ranked. Therefore, TSVD can be employed and a new set of equivalent sources can be constructed by a linear combination of the original CSBs. It is proved that the radiation power of the new sources is proportional to the square of the corresponding singular values. This provides a theoretical guideline to drop the insignificant singular vectors in the calculation. In doing so, the efficiency of the original NCSB method can be much improved while a reasonably good accuracy is maintained. Several numerical tests are conducted to validate the proposed method.
\end{abstract}

\section{Introduction}

Integral equation (IE) method has been intensively studied in the analysis of electromagnetic (EM) radiation and scattering in recent years. For a numerical solution, the IE is usually discretized into a matrix equation by the method of moments (MoM) [1]. In order to conduct large-scale simulations, a variety of numerical techniques have been developed based on MoM. These techniques are carried out efficiently either by reduction of the far-field interaction exploiting the physical or mathematical properties of the IE or by using well-designed basis/test functions to reduce the total number of unknowns.

In the first category, the multilevel fast multipole algorithm (MLFMA) [2] is widely used, where the far-field interactions can be accelerated by aggregation, translation, and disaggregation operations. Besides it, the low rank property of the MoM far-field submatrices leads to a series of matrix decomposition methods, such as the multilevel matrix decomposition algorithm (MLMDA) [3] and the adaptive cross approximation (ACA) [4, 5]. For the second category, the higher order basis functions (HOBF) [6], phase extracted basis functions (PE) $[7,8]$, characteristic basis function (CBF) $[9,10]$, or body of revolution (BoR) [11] MoM are proposed to reduce the number of unknowns in a given problem.

Following the first category, a complex source beammethod of moments (CSB-MoM) is recently proposed to accelerate the far-field interactions of MoM [12]. In this method, the object is divided into groups and complex source beams (CSBs) [13] are used to expand the fields of the basis functions residing in each group [14, 15]. Hence, the far-field interactions of these basis/test functions can be accounted for by their equivalent CSB expansions. Due to the directional nature of CSBs [16], the interactions usually involve only a small portion of the total CSBs. A multilevel version of this method is developed in [17] to further improve the computational efficiency. The branch cut issue of CSBs, which might degrade the accuracy in the evaluation of group interactions, can be avoided by a proper choice of the CSB parameters. 
Unfortunately, in this method, the CSB expansions at any level are calculated directly from the basis functions contained in the finest level. This operation is very computationally expensive. Hence, the application of this method to electrically large problems is prohibited. To overcome this difficulty, a nested complex source beam (NCSB) method is proposed by utilizing an equivalent relationship between adjacent levels [18]. This relationship is built by treating CSBs in the child group as new sources and applying the far-field matching to get their CSB expansions in the parent group. In doing this, the computational complexity of NCSB can be reduced to $O(N \log N)$, where $N$ is the number of unknowns. However, it should be noted that the CSBs involved in the translation process have to be determined empirically. Moreover, the aggregation and disaggregation matrices are still in a dense format.

To fully exploit the directional property of CSBs, a truncated singular value decomposition (TSVD) method is applied to compress the aggregation, translation, and disaggregation matrices of NCSB in this paper. After SVD, a set of equivalent sources can be obtained by a linear combination of the original CSBs. A theoretical proof reveals that the radiation power of the new sources is proportional to the square of the corresponding singular values. This provides a guideline for the truncation in the calculation. Thereby, the proposed method not only leads to a significant improvement of the computational efficiency but also provides a flexible compromise between accuracy and computational cost.

\section{Formulations}

Given a 3D perfectly electrical conducting (PEC) body defined by its surface, a MoM matrix equation can be obtained as

$$
\mathbf{Z I}=\mathbf{Z}_{N} \mathbf{I}+\mathbf{Z}_{F} \mathbf{I}=\mathbf{V},
$$

where $\mathbf{I}$ is the unknown vector containing the expansion coefficients of the current, $\mathbf{V}$ is the excitation vector, and $\mathbf{Z}$ is the MoM impedance matrix. After grouping, this dense matrix can be separated into the near-field interaction part $\mathbf{Z}_{N}$ and the far-field interaction counterpart $\mathbf{Z}_{F}$.

In CSB-MoM, the far-field interactions between different groups are carried out by a series of CSBs launched on a complex equivalence surface enclosing each group [14]. The far-field part of the matrix-vector product (MVP) in CSBMoM can be represented as

$$
\mathbf{Z}_{F} \mathbf{I}=\mathbf{Z}_{m m^{\prime}} \mathbf{I}_{m^{\prime}}=\sum_{p, p^{\prime}=\theta, \phi} \mathbf{Y}_{m}^{p} \mathbf{T}_{L, m m^{\prime}}^{p p^{\prime}} \mathbf{W}_{m^{\prime}}^{p^{\prime}} \mathbf{I}_{m^{\prime}},
$$

where $m$ and $m^{\prime}$ denote the observation and source groups and $L$ indicates the finest level (single level in CSB-MoM). $\mathbf{W}_{m^{\prime}}^{p^{\prime}}$ and $\mathbf{Y}_{m}^{p}$ are, respectively, the expansion matrix and local expansion matrix for both $\theta$ and $\phi$ components. The farfield matching technique can be used for constructing $\mathbf{W}_{m^{\prime}}^{p^{\prime}}$ and $\mathbf{Y}_{m}^{p}$ in electric field integral equation (EFIE), magnetic field integral equation (MFIE) [19], and PMCHWT integral equation [20]. $\mathbf{T}_{L, m m^{\prime}}^{p p^{\prime}}$ is the translation matrix, of which the elements are expressed as

$$
\left[\mathbf{T}_{L, m m^{\prime}}^{p p^{\prime}}\right]_{q q^{\prime}}=\widehat{p}_{q} \cdot \overline{\bar{G}}\left(\widetilde{\mathbf{r}}_{L, m}^{q} \mid \widetilde{\mathbf{r}}_{L, m^{\prime}}^{q^{\prime}}\right) \cdot \widehat{p}_{q^{\prime}}^{\prime}
$$

where $\widehat{p}_{q}$ and $\widehat{p}_{q^{\prime}}^{\prime}$ denote the unit vectors of $q$ th and $q^{\prime}$ th CSB, respectively. The complex position vectors $\widetilde{\mathbf{r}}_{L, m}^{q}$ and $\widetilde{\mathbf{r}}_{L, m^{\prime}}^{\prime q^{\prime}}$ are the launch points of CSBs. $\overline{\bar{G}}\left(\widetilde{\mathbf{r}}_{m}^{q} \mid \widetilde{\mathbf{r}}_{m^{\prime}}^{\prime q^{\prime}}\right)$ is the dyadic Green function with complex arguments.

By using this representation, the CSB expansion coefficients for the source group $m^{\prime}$ can be expanded from the surface currents as

$$
\mathbf{S}_{L, m^{\prime}}=\left[\begin{array}{l}
\mathbf{S}_{L, m^{\prime}}^{\theta} \\
\mathbf{S}_{L, m^{\prime}}^{\phi}
\end{array}\right]=\left[\begin{array}{c}
\mathbf{W}_{m^{\prime}}^{\theta} \\
\mathbf{W}_{m^{\prime}}^{\phi}
\end{array}\right] \mathbf{I}_{m^{\prime}}
$$

On the other hand, the CSB expansion coefficients $\mathbf{R}_{L, m^{\prime}}$ for the receiving group $m$ are translated from the transmitting group $m^{\prime}$ :

$$
\mathbf{R}_{L, m^{\prime}}=\mathbf{T}_{L, m m^{\prime}}^{p p^{\prime}} \mathbf{S}_{L, m^{\prime}}
$$

As has been mentioned previously, the directional property of CSBs can be used to reduce the computational cost in the translation procedure. The translation window can be set based on numerical experiments for different group sizes and CSB parameters, as is done in [18].

2.1. NCSB Formulations. Motivated by the idea of MLFMA [2], CSB-MoM can be naturally extended to a multilevel version if a similar octree data structure is adopted. However, the multilevel CSB or MLCSB proposed in [17] calculates the CSB expansion coefficients for each level directly from the basis functions in the finest level. The lack of a proper mechanism of interlevel aggregation and disaggregation prohibits this method from analyzing large-unknown problems. To end this, the NCSB is proposed in [18] to introduce the aggregation and disaggregation operations between every two adjacent levels through a proper far-field matching.

In the following, we obtain the aggregation matrix in a slightly different way by further utilizing the directional property of the radiating far field of CSBs. Moreover, the symmetry is fully exploited so that only one aggregation matrix is needed for all the eight child groups of one parent group. First, a linear system is set up to build the equivalent relationship of CSBs between two adjacent levels:

$$
\mathbf{Z}_{l} \mathbf{A}_{l c}=\mathbf{F}_{l+1, c} \quad c=1,2, \ldots, 8,
$$

where $\mathbf{Z}_{l}$ is the matching matrix that connects the equivalent CSB sources with the far fields $\mathbf{F}_{l+1, c}$. The expression of the matching matrix is the same as the matrix used for CSB expansion with electric current type equivalent sources in CSB-MoM $[12,14]$. Different from CSB-MoM of single level, the equivalent CSB sources in $\mathbf{Z}_{l}$ are launched in level $l$, and 
the far fields here are radiated by CSBs in child level $l+1$ with both $\theta$ and $\phi$ components:

$$
\mathbf{F}_{l+1, c}=\left[\begin{array}{ll}
\mathbf{E}_{l+1, c}^{\theta \theta} & \mathbf{E}_{l+1, c}^{\theta \phi} \\
\mathbf{E}_{l+1, c}^{\phi \theta} & \mathbf{E}_{l+1, c}^{\phi \phi}
\end{array}\right],
$$

where the elements in each block matrix are

$$
\left[\mathbf{E}_{l+1, c}^{p^{\prime} p}\right]_{q_{l} q_{l+1}}=\widehat{p}_{q_{l}}^{\prime} \overline{\bar{G}}\left(\mathbf{r}_{q_{l}} \mid \widetilde{\mathbf{r}}_{q_{l+1}, c}^{\prime}\right) \widehat{p}_{q_{l+1}} .
$$

It is noted that, for a parent group in the octree, there are eight different child groups at most. Hence, eight corresponding aggregation matrices are required in the calculation. However, by fully exploiting the symmetry property, we discover that only one aggregation matrix is needed and others can be easily deduced from it. In the following, the subscript $c$ will be omitted for simplicity.

In our method, the radiating far fields on the righthand-sides of (6) can be fast calculated by truncating CSB directional fields within the paraxial regions. Once $\mathbf{Z}_{l}$ and $\mathbf{F}_{l+1, \mathrm{c}}$ are assembled, the aggregation matrix for level $l$

$$
\mathbf{A}_{l}=\left[\begin{array}{ll}
\widetilde{\mathbf{A}}_{l}^{\theta \theta} & \widetilde{\mathbf{A}}_{l}^{\theta \phi} \\
\widetilde{\mathbf{A}}_{l}^{\phi \theta} & \widetilde{\mathbf{A}}_{l}^{\phi \phi}
\end{array}\right]_{2 \mathrm{Q}_{l} \times 2 \mathrm{Q}_{l+1}}
$$

can be numerically solved. Here $Q_{l}$ and $Q_{l+1}$ are the numbers of CSBs in levels $l$ and $l+1$. In this work, we apply the least square method to improve the stability of the solution. In (9), the element $\left[\widetilde{\mathbf{A}}_{l}^{p^{\prime} p^{\prime \prime}}\right]_{q_{l} q_{l+1}}$ in the block aggregation matrix is the mapping coefficient between the $q_{l+1}$ th CSB in the child group and the $q_{l}$ th CSB in its parent group. By using this relationship, the disaggregation matrix can be easily obtained from the transpose of the aggregation matrix. The CSB expansion coefficients of the parent group in level $l$ can be obtained efficiently from its child groups in level $l+1$ with the aggregation matrix:

$$
\mathbf{S}_{l, m^{\prime}}=\left[\begin{array}{l}
\mathbf{S}_{l, m^{\prime}}^{\theta} \\
\mathbf{S}_{l, m^{\prime}}^{\phi}
\end{array}\right]=\sum_{n^{\prime} \in \operatorname{child}\left(m^{\prime}\right)} \mathbf{A}_{l} \mathbf{S}_{l+1, n^{\prime}} .
$$

Similar to MLFMA, the CSB expansion coefficients of a receiving group in level $l+1$ is obtained from both of the translation in the same level and the disaggregation from its parent level $l$. Therefore, we have

$$
\mathbf{R}_{l+1, m}=\left[\begin{array}{l}
\mathbf{R}_{l+1, m}^{\theta} \\
\mathbf{R}_{l+1, m}^{\phi}
\end{array}\right]=\mathbf{A}_{l}^{T}\left[\begin{array}{l}
\mathbf{R}_{l, n}^{\theta} \\
\mathbf{R}_{l, n}^{\phi}
\end{array}\right]=\mathbf{A}_{l}^{T} \mathbf{R}_{l, n} .
$$

As has been elaborated in the above, the NCSB algorithm can be constructed in much the same way as MLFMA. However, all the operation matrices involved in NCSB are dense matrices, in contrast with the diagonal matrices in MLFMA. Improvement can be made by utilizing the unique property of CSBs. Specifically, all the CSBs are directional; that is to say, a CSB can only directionally interact with another CSB in the far-field region. Hence, the dense aggregation/disaggregation and translation matrices are usually low-ranked. To take advantage of the directional property of CSBs, TSVD will be applied to compress these matrices in the following sections.
2.2. TSVD in Aggregation/Disaggregation Process. For the aggregation matrix in (9), the SVD factorization can be applied as

$$
\mathbf{A}_{l}=\mathbf{U}_{l}^{A} \mathbf{\Sigma}_{l}^{A}\left(\mathbf{V}_{l}^{A}\right)^{H}
$$

where $\left[\mathbf{U}_{l}^{A}\right]_{2 Q_{l} \times 2 Q_{l}}$ and $\left[\mathbf{V}_{l}^{A}\right]_{2 Q_{l+1} \times 2 Q_{l+1}}$ are unitary matrices and $\Sigma_{l}^{A}=\operatorname{diag}\left\{\sigma_{1}, \sigma_{2}, \ldots, \sigma_{2 \mathrm{Q}_{l+1}}\right\}_{2 \mathrm{Q}_{l} \times 2 \mathrm{Q}_{l+1}}$ is a diagonal matrix whose elements are the nonnegative real singular values listed in a descending order. The columns of $\mathbf{V}_{l}^{A}$ represent a new set of orthonormal child CSB modes in level $l+1$, which are obtained as linear combinations of the original CSBs. Matrix $\mathbf{U}_{l}^{A}$ defines a complete set of orthonormal parent CSB modes associated with the child modes. Diagonal matrix $\Sigma_{l}^{A}$ maps the child modes to the corresponding parent modes with weighting of the singular values.

The physical meaning of the above SVD can be interpreted as follows. One set of the child CSBs is coupled to another set of parent CSBs, with the coupling strength governed by the corresponding singular value. Hence, after $\mathrm{SVD}$, the aggregation procedure is converted from beam-tobeam coupling into mode-to-mode coupling. Furthermore, we can prove that the radiated power by each child CSB mode is proportional to the square of the singular value (see Appendix). This power-related mode provides a helpful guideline for extracting the most significant part in the aggregation matrix.

If $r_{l}$ is the effective rank of $\mathbf{A}_{l}$ for a prescribed threshold $\epsilon$, the SVD decomposition in (12) can be truncated and approximated as

$$
\mathbf{A}_{l} \simeq \widetilde{\mathbf{U}}_{l}^{A} \widetilde{\mathbf{\Sigma}}_{l}^{A}\left(\widetilde{\mathbf{V}}_{l}^{A}\right)^{H}
$$

where $\widetilde{\boldsymbol{\Sigma}}_{l}^{A}$ is formed by the first (largest) $r_{l}$ singular values in $\Sigma_{l}^{A}$ satisfying $\sigma_{r_{l}+1}<\epsilon \sigma_{1} . \widetilde{\mathbf{U}}_{l}^{A}$ and $\widetilde{\mathbf{V}}_{l}^{A}$ denote the corresponding submatrices consisting of the first $r_{l}$ columns of $\mathbf{U}_{l}^{A}$ and $\mathbf{V}_{l}^{A}$, respectively. Hence, $\widetilde{\mathbf{V}}_{l}^{A}$ contains all the child CSB modes having radiated power higher than $\epsilon^{2} \sigma_{1}^{2}$. According to the prescribed accuracy, this reduced set of CSBs in child group suffices to represent the far field of the parent group. Since disaggregation is implemented as the transpose of aggregation, the TSVD procedure of disaggregation matrix can be implemented by fully utilizing $\widetilde{\mathbf{U}}_{l}^{A}$ and $\widetilde{\mathbf{V}}_{l}^{A}$ in (13).

2.3. TSVD in Translation Process. As the CSB decays rapidly in the directions orthogonal to the beam propagation axis, in the translation process, the interactions between farfield groups are carried out by CSBs whose beam axis are alined with the line connecting the group centers. These CSBs can be easily selected by setting a conical truncation window. To avoid amount of experiments needed to determine the minimal conical angle, in this paper, we first set the truncation angle when the field of a CSB decays to $-20 \mathrm{~dB}$ of the aforementioned prescribed accuracy times the maximum value. According to this criterion, the CSB selection matrices $\mathbf{L}_{l, m m^{\prime}}^{\prime}$ and $\mathbf{L}_{l, m m^{\prime}}$ are introduced to pick up 
TABLE 1: Computational statistics.

\begin{tabular}{|c|c|c|c|c|c|c|c|c|}
\hline Method & $\begin{array}{c}Q_{l} \\
\text { (level 2-4) }\end{array}$ & $\begin{array}{l}\text { Aggregation TSVD } \\
\text { rank (level 2-3) }\end{array}$ & $\begin{array}{c}K_{l} \\
\text { (level 2-4) }\end{array}$ & $\begin{array}{c}\text { Translation TSVD } \\
\text { rank (level 2-4) }\end{array}$ & $\begin{array}{c}\text { Aggregation \& } \\
\text { Translation Mem. }\end{array}$ & $\begin{array}{l}\text { Setup } \\
\text { time }\end{array}$ & $\begin{array}{l}\text { Solving } \\
\text { time }\end{array}$ & $\begin{array}{l}\text { Total } \\
\text { time }\end{array}$ \\
\hline NCSB & \multirow{4}{*}{$5738 / 1758 / 682$} & - & \multirow{4}{*}{$1213 / 681 / 289$} & - & $19230.5 \mathrm{MB}$ & $511 \mathrm{~s}$ & $3864 s$ & $4375 s$ \\
\hline$\epsilon=10^{-2}$ & & $635 / 509$ & & $59 / 48 / 66$ & $1429.9 \mathrm{MB}$ & $936 \mathrm{~s}$ & $1178 \mathrm{~s}$ & $2114 \mathrm{~s}$ \\
\hline$\epsilon=10^{-3}$ & & $1154 / 730$ & & $119 / 131 / 108$ & $3119.3 \mathrm{MB}$ & $958 \mathrm{~s}$ & $1345 \mathrm{~s}$ & $2303 \mathrm{~s}$ \\
\hline$\epsilon=10^{-4}$ & & $1600 / 919$ & & $206 / 199 / 142$ & $5548.7 \mathrm{MB}$ & $992 \mathrm{~s}$ & $1488 \mathrm{~s}$ & $2480 \mathrm{~s}$ \\
\hline
\end{tabular}

the most significant CSBs in the transmitting and receiving groups; namely,

$$
\begin{aligned}
& {\left[\mathbf{T}_{l, m m^{\prime}}^{p p^{\prime}}\right]_{Q_{l} \times Q_{l}}} \\
& \quad=\left[\mathbf{L}_{l, m m^{\prime}}\right]_{Q_{l} \times K_{l}}\left[\widetilde{\mathbf{T}}_{l, m m^{\prime}}^{p p^{\prime}}\right]_{K_{l} \times K_{l}}\left[\mathbf{L}_{l, m m^{\prime}}^{\prime}\right]_{K_{l} \times \mathrm{Q}_{l}},
\end{aligned}
$$

where only one element in each row (column) of $\mathbf{L}_{l, m m^{\prime}}^{\prime}$ $\left(\mathbf{L}_{l, m m^{\prime}}\right)$ is 1 , if the corresponding CSB is within the conical truncation window; otherwise they are set to 0 .

After this truncation based on the beam width of CSBs, the dimension of the translation matrix $\widetilde{\mathbf{T}}_{l, \mathrm{~mm}^{\prime}}^{p p^{\prime}}$ is reduced from $Q_{l} \times Q_{l}$ to $K_{l} \times K_{l}$. However, it is still rank-deficient. The TSVD procedure is then applied to this reduced translation matrix to gain further compression:

$$
\widetilde{\mathbf{T}}_{l, m m^{\prime}}^{p p^{\prime}} \simeq \widetilde{\mathbf{U}}_{l, m m^{\prime}}^{T, p p^{\prime}} \widetilde{\mathbf{\Sigma}}_{l, m m^{\prime}}^{T, p p^{\prime}}\left[\widetilde{\mathbf{V}}_{l, m m^{\prime}}^{T, p p^{\prime}}\right]^{H},
$$

where $\widetilde{\mathbf{U}}_{l, m m^{\prime}}^{T, p p^{\prime}}, \widetilde{\boldsymbol{\Sigma}}_{l, m m^{\prime}}^{T, p p^{\prime}}$, and $\widetilde{\mathbf{V}}_{l, m m^{\prime}}^{T, p p^{\prime}}$ are defined similarly as in (13), except that the threshold is set as $\epsilon^{2}$ instead of $\epsilon$ to account for the effects from both of the transmitting and receiving groups.

The proposed TSVD procedure is effective by providing new set of CSB modes based on the singular vectors. The significance in the total radiated power is governed by the descending singular values. The SVD process fully exploits the spatial window property of CSBs, which is independent of the geometry, current distribution, and types of IE. By defining an error threshold, redundant modes which contribute negligibly to the radiated power are excluded. Therefore, the aggregation, translation, and disaggregation matrices can be represented in a very compact form. Furthermore, the balance between accuracy and computational efficiency can be easily controlled by adjusting the truncation threshold.

\section{Numerical Results}

In this section, several numerical tests are conducted to demonstrate the efficiency and validity of the proposed method.

Firstly, the low rank property of the aggregation matrices of NCSB method is studied. Figure 1 shows the normalized singular values of the aggregation matrices for level 2 (with electrical size of $8 \lambda$ and aggregating from level 3 ) and level 3 (with electrical size of $4 \lambda$ and aggregating from level 4 ). The

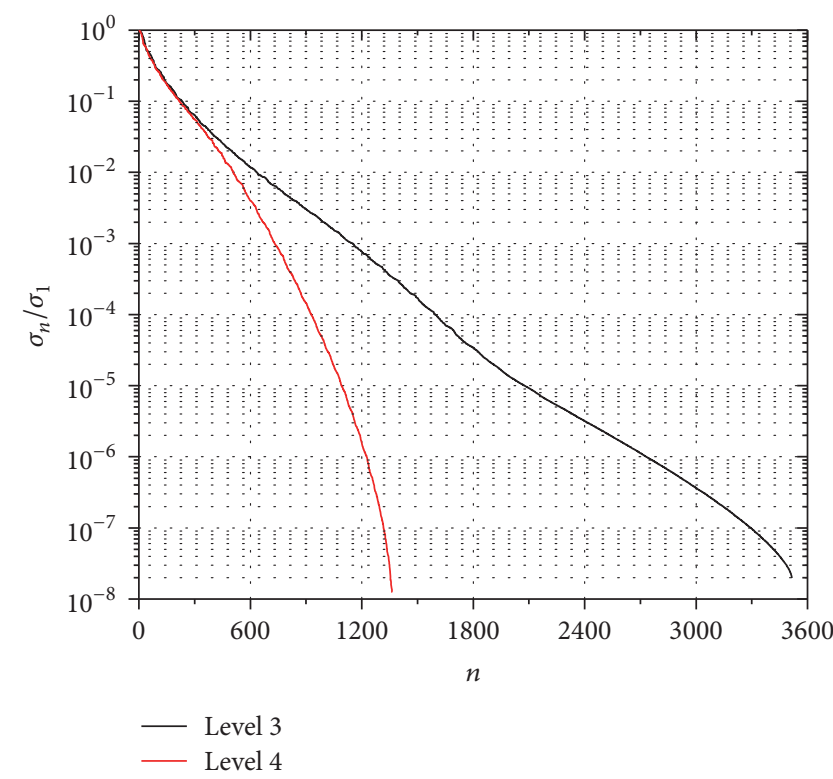

FIGURE 1: Normalized singular values of the aggregation matrix.

numbers of CSBs for levels 2, 3, and 4 are 5738, 1758, and 682, as listed in Table 1 . Considering both $\theta$ and $\phi$ components of CSBs, the dimensions of the aggregation matrices for level 2 and level 3 are $11476 \times 3516$ and $3516 \times 1364$, respectively. As shown in Figure 1, the singular values for both matrices decrease rapidly, indicating a quick decay of the radiated power from individual child CBS mode. Due to this fact, the aggregation matrices are truncated by thresholds of $\epsilon=10^{-2}$, $10^{-3}$, and $10^{-4}$ for comparison. Figure 2 depicts the relative errors of the radiated far field (an effective measure of the aggregation error) with respect to the observation angles. In particular, this relative error is defined as

$$
e(\phi)=\frac{\left\|\mathbf{E}(\phi)-\mathbf{E}_{\mathrm{agg}}(\phi)\right\|_{2}}{\|\mathbf{E}(\phi)\|_{2}}
$$

and here $\mathbf{E}(\phi)$ is the reference field, $\mathbf{E}_{\mathrm{agg}}(\phi)$ is the field obtained via aggregations by TSVD, and $\phi$ is the observation angle which is set on a azimuth circle. In Figure 2, the reference field is radiated directly by the CSBs of a group with the size of $2 \lambda$ in the fourth level. Then two aggregation steps with TSVD are performed to this group until the second level. Finally, the field $\mathbf{E}_{\mathrm{agg}}(\phi)$ is calculated by the CSBs in the second level. Figure 2 shows that the relative aggregation error can be effectively controlled by the TSVD threshold $\epsilon$. 


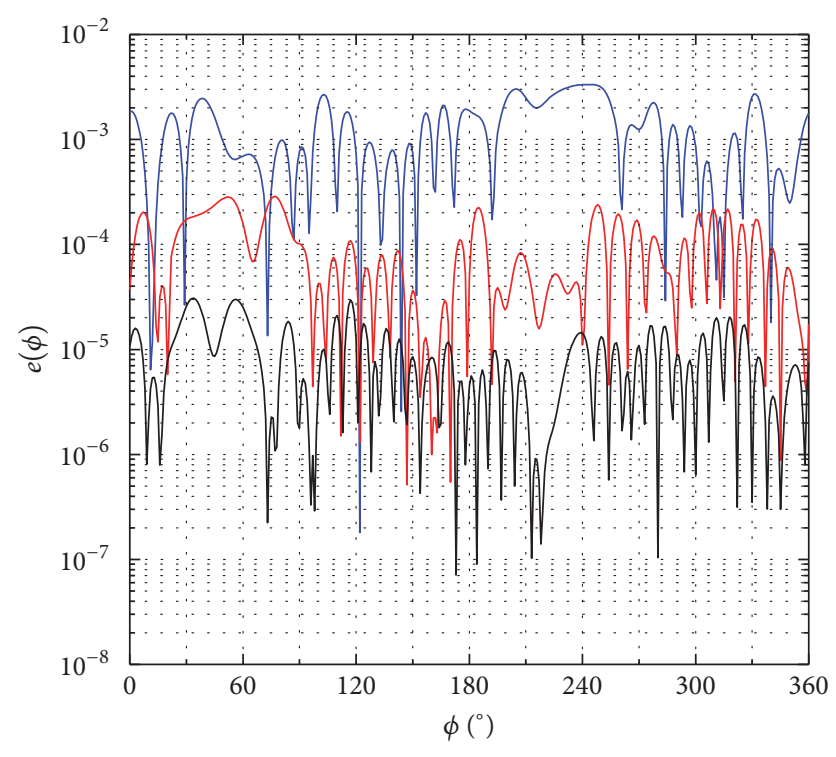

NCSB-TSVD $\varepsilon=10^{-2}-$ NCSB-TSVD $\varepsilon=10^{-3}$

FIGURE 2: Relative aggregation errors measured by the radiated far field.

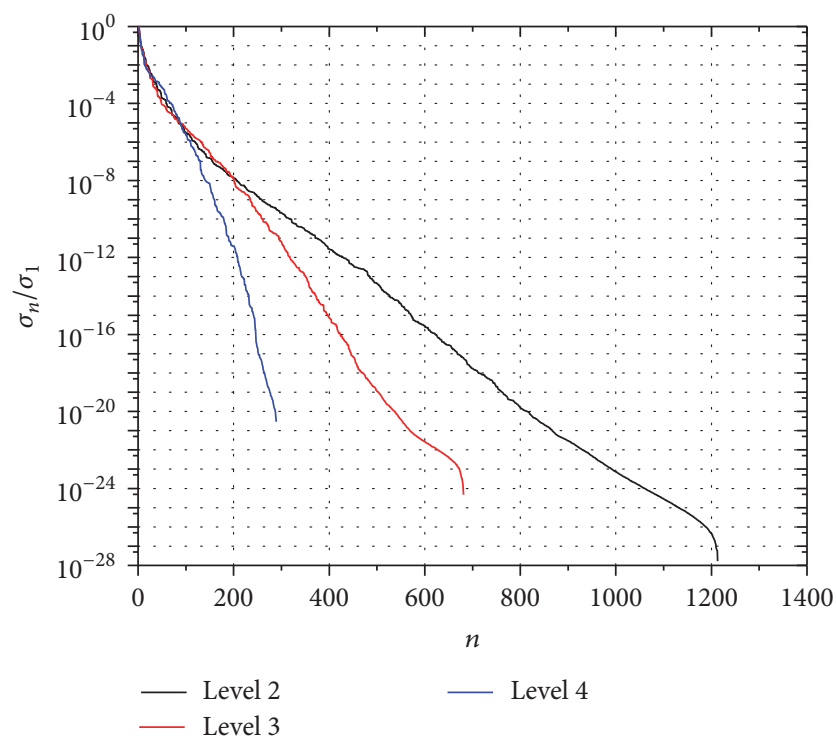

FIGURE 3: Normalized singular values of the reduced translation matrix.

Next, we investigate the TSVD based translation process. As shown in Table 1, after the aforementioned window selection, the number of CSBs for the reduced translation $\left(K_{l}\right)$ drops to 1213, 681, and 289 for levels 2, 3, and 4, respectively. However, the information of the reduced translation matrices is still redundant, and the effective rank can be further compressed. Figure 3 shows the normalized singular values of the reduced translation matrices, where a rapid decrease can again be observed for individual CSB mode. As has been explained, the truncation thresholds for translation matrices

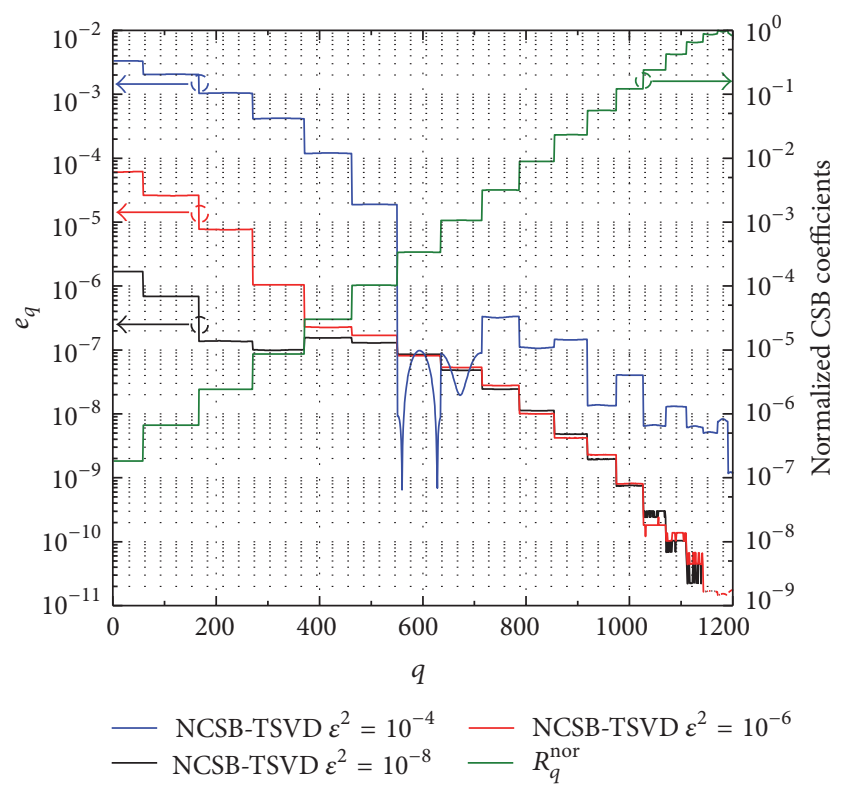

FiguRE 4: Relative translation errors.

should be set as $\epsilon^{2}$, namely, $10^{-4}, 10^{-6}$, and $10^{-8}$, in this example. The relative translation error

$$
e_{q}=\frac{\left|R_{q}^{\mathrm{tsvd}}-R_{q}\right|}{\left|R_{q}\right|} \quad q=1,2, \ldots, K_{l}
$$

is depicted in Figure 4, where $R_{q}$ and $R_{q}^{\text {tsvd }}$ are the CSB expansion coefficients in level 2 , translated by the original translation and the TSVD counterpart, respectively. Even though the error is relatively higher for small indices of CSBs, the corresponding normalized expansion coefficients $R_{q}^{\text {nor }}=$ $\left|R_{q}\right| / \max _{j}\left|R_{j}\right|$ are also relatively small. Hence, these CSBs will contribute to the total field insignificantly. In other words, TSVD maintains reasonably good translation accuracy for both the strongly coupled CSBs (with larger indices) and the weakly coupled CSBs (with smaller indices).

Finally, to demonstrate the overall performance of the proposed method, the scattering problem of a diameter $32 \lambda$ sphere is presented. It is illuminated by an incident plane wave $\left(\theta_{i}=0^{\circ}, \varphi_{i}=0^{\circ}\right)$ at $300 \mathrm{MHz}$ and solved by combined field integral equation (CFIE). Figure 5 shows the bistatic RCS results compared with the analytical solution of Mie series from $120^{\circ}$ to $180^{\circ}$. We can see a very good agreement is obtained for $\epsilon=10^{-3}$ and $\epsilon=10^{-4}$. Furthermore, a good agreement is still obtained for $\epsilon=10^{-2}$. To quantify the accuracy, the RMS value of the error $E=\mid \delta_{\text {NCSB-TSVD }}-$ $\left.\delta_{\text {Mie }}|/| \delta_{\text {Mie }}\right|_{\max }$ has been calculated, $\delta$ being the RCS $\left(\mathrm{m}^{2}\right)$. RMS errors of $0.18 \%, 0.0806 \%$, and $0.0768 \%$ are found for the thresholds $10^{-2}, 10^{-3}$, and $10^{-4}$. At the same time, the computational efficiency can be significantly improved by the proposed method. The statistics are summarized in Table 1. It can be observed that a great computational improvement is obtained in terms of CPU time and memory consumption. It is also shown that a good compromise between accuracy and the computational efficiency can be easily achieved, by a proper choice of the truncation parameter. 


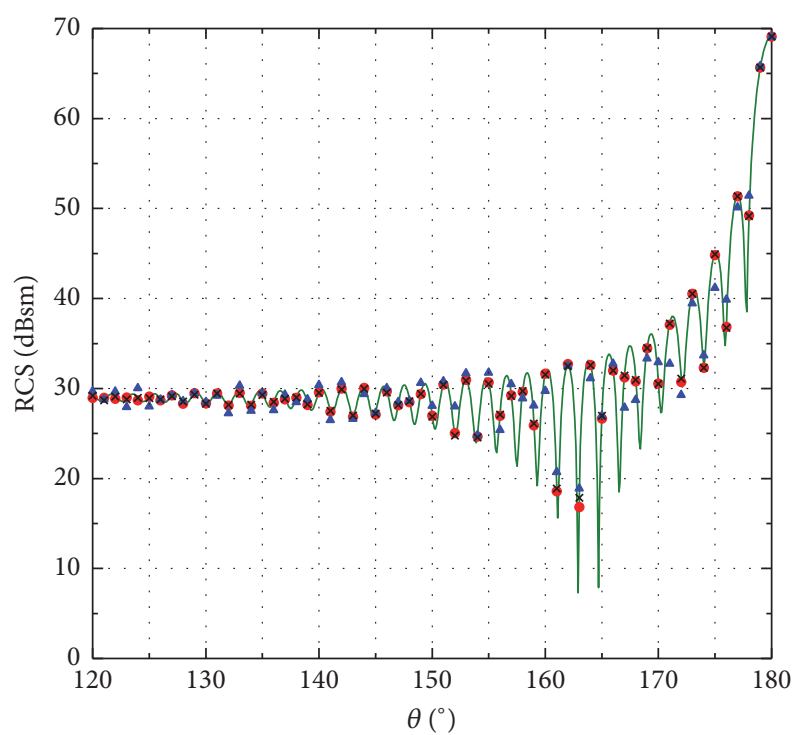

$\triangle \operatorname{NCSB}-T S V D \varepsilon=10^{-2} \quad$ NCSB-TSVD $\varepsilon=10^{-3}$

$$
\times \operatorname{NCSB}-T S V D \varepsilon=10^{-4} \longrightarrow \text { Mie }
$$

FIGURE 5: Bistatic RCS of a PEC sphere with a diameter $32 \lambda$, by NCSB-TSVD and Mie-series solution.

\section{Conclusion}

In this paper, we have proposed an efficient implementation of NCSB. Different from previous work, the aggregation process is first constructed by the truncated far-field matching. The dimension of the translation matrix is then reduced based on the beam width of the CSBs. Finally, TSVD is used to compress the aggregation, translation, and disaggregation matrices by fully exploiting the directional property of CSBs. It is shown that the radiated power of the new sources from SVD is proportional to the square of the corresponding singular values. This power-dependent mode provides a theoretical guideline for extracting the most significant CSB contributions in the calculation. Therefore, the desired balance between accuracy and computational efficiency can be easily controlled by adjusting the truncation threshold.

\section{Appendix}

The radiated power by the $q$-th CSB expansion coefficient of group $m$ in level $l$ can be calculated by an integral on the matching surface as:

$$
\begin{aligned}
P_{m}^{q} & =\frac{1}{2 \eta} \int_{s} \mathbf{E}(r) \cdot \mathbf{E}^{*}(r) d s \\
& =\frac{1}{2 \eta} \int_{s} \sum_{p, p^{\prime}=\theta, \phi}\left[E_{l, m}^{p^{\prime} p}(r) S_{q}^{p}\right]\left[E_{l, m}^{p^{\prime} p}(r) S_{q}^{p}\right]^{*} d s \\
& \propto \sum_{p=\theta, \phi} S_{q}^{p}\left(S_{q}^{p}\right)^{*},
\end{aligned}
$$

where $S_{q}^{p}=\left[\mathbf{S}_{l, m}^{p}\right]_{q}$. Therefore, the total radiated power can be obtained by summing up the contributions from all CSBs:

$$
P_{m}=\sum_{q=1}^{\mathrm{Q}_{l+1}} P_{m}^{q} \propto \sum_{q=1}^{\mathrm{Q}_{l+1}} \sum_{p=\theta, \phi} S_{q}^{p}\left(S_{q}^{p}\right)^{*},
$$

which can be further expressed in matrix form as:

$$
P_{m} \propto \mathbf{S}_{l, m}^{H} \mathbf{S}_{l, m}
$$

Sine the CSB expansion coefficient can be aggregated from the child groups:

$$
\mathbf{S}_{l, m}=\mathbf{A}_{l} \mathbf{S}_{l+1, n}
$$

we have

$$
\mathbf{S}_{l, m}^{H} \mathbf{S}_{l, m}=\mathbf{S}_{l+1, n}^{H} \mathbf{V}_{l}^{A}\left(\boldsymbol{\Sigma}_{l}^{A}\right)^{2}\left(\mathbf{V}_{l}^{A}\right)^{H} \mathbf{S}_{l+1, n} .
$$

By defining the new child CSB modes as $\mathbf{S}_{l+1, n}^{\prime}=\left(\mathbf{V}_{l}^{A}\right)^{H} \mathbf{S}_{l+1, n}$, we obtain

$$
\mathbf{S}_{l, m}^{H} \mathbf{S}_{l, m}=\mathbf{S}_{l+1, n}^{\prime H}\left(\Sigma_{l}^{A}\right)^{2} \mathbf{S}_{l+1, n}^{\prime} .
$$

Substituting (A.6) into (A.3) leads to

$$
P_{m} \propto \mathbf{S}_{l+1, n}^{\prime H}\left(\mathbf{\Sigma}_{l}^{A}\right)^{2} \mathbf{S}_{l+1, n}^{\prime}
$$

which can be rewritten as

$$
P_{m} \propto \sum_{q=1}^{\mathrm{Q}_{l+1}} \sigma_{i}^{2}\left|\left[\mathbf{s}_{l+1, n}^{\prime}\right]_{q}\right|^{2}
$$

This result shows that the total radiated power by CSBs is proportional to the square of singular value corresponding to the child mode.

\section{Conflicts of Interest}

The authors declare that there are no conflicts of interest regarding the publication of this paper.

\section{Acknowledgments}

This work is supported partly by the Programme of Introducing Talents of Discipline to Universities under Grant no. b07046, the National Excellent Youth Fund by NSFC no. 61425010, and the National Science Foundation of China under Grant no. 61490695.

\section{References}

[1] R. F. Harrington and J. L. Harrington, Field computation by moment methods, Oxford University Press, 1996.

[2] O. Ergül and L. Gürel, The Multilevel Fast Multipole Algorithm (MLFMA) for Solving Large-Scale Computational Electromagnetics Problems, John Wiley \& Sons, 2014. 
[3] J. M. Rius, J. Parrón, E. Úbeda, and J. R. Mosig, "Multilevel matrix decomposition algorithm for analysis of electrically large electromagnetic problems in 3-D," Microwave and Optical Technology Letters, vol. 22, no. 3, pp. 177-182, 1999.

[4] K. Zhao, M. N. Vouvakis, and J.-F. Lee, "The adaptive cross approximation algorithm for accelerated method of moments computations of EMC problems," IEEE Transactions on Electromagnetic Compatibility, vol. 47, no. 4, pp. 763-773, 2005.

[5] D. Z. Ding, Y. Shi, Z. N. Jiang, and R. S. Chen, "Augmented EFIE with adaptive cross approximation algorithm for analysis of electromagnetic problems," International Journal of Antennas and Propagation, vol. 2013, Article ID 487276, 9 pages, 2013.

[6] X.-M. Pan, L. Cai, and X.-Q. Sheng, "An efficient high order multilevel fast multipole algorithm for electromagnetic scattering analysis," Progress In Electromagnetics Research, vol. 126, pp. 85-100, 2012.

[7] Z.-P. Nie, S. Yan, S. He, and J. Hu, "On the basis functions with traveling wave phase factor for efficient analysis of scattering from electrically large targets," Progress In Electromagnetics Research, vol. 85, pp. 83-114, 2008.

[8] S. Yan, S. Ren, Z. Nie, S. He, and J. Hu, "Efficient analysis of electromagnetic scattering from electrically large complex objects by using phase-extracted basis functions," IEEE Antennas and Propagation Magazine, vol. 54, no. 5, pp. 88-108, 2012.

[9] V. V. S. Prakash and R. Mittra, "Characteristic basis function method: a new technique for efficient solution of method of moments matrix equations," Microwave and Optical Technology Letters, vol. 36, no. 2, pp. 95-100, 2003.

[10] H. Shao, J. Hu, W. Lu, H. Guo, and Z. Nie, "Analyzing largescale arrays using tangential equivalence principle algorithm with characteristic basis functions," Proceedings of the IEEE, vol. 101, no. 2, pp. 414-422, 2013.

[11] Y. Li, J. Hu, and Z. Nie, "Solving scattering from multiple bodies of revolution by modal characteristic basis function method with sparse matrix technique," IEEE Antennas and Wireless Propagation Letters, vol. 15, pp. 806-809, 2016.

[12] K. Tap, P. H. Pathak, and R. J. Burkholder, "Complex source beam-moment method procedure for accelerating numerical integral equation solutions of radiation and scattering problems," Institute of Electrical and Electronics Engineers. Transactions on Antennas and Propagation, vol. 62, no. 4, part 2, pp. 2052-2062, 2014.

[13] T. B. Hansen and G. Kaiser, "Huygens' principle for complex spheres," Institute of Electrical and Electronics Engineers. Transactions on Antennas and Propagation, vol. 59, no. 10, pp. 38353847, 2011.

[14] K. Tap, P. H. Pathak, and R. J. Burkholder, "Exact complex source point beam expansions for electromagnetic fields," IEEE Transactions on Antennas and Propagation, vol. 59, no. 9, pp. 3379-3390, 2011.

[15] E. Martini and S. Maci, "Generation of complex source point expansions from radiation integrals," Progress in Electromagnetics Research, vol. 152, pp. 17-31, 2015.

[16] G. A. Deschamps, "Gaussian beam as a bundle of complex rays," Electronics Letters, vol. 7, no. 23, pp. 684-685, 1971.

[17] Z. H. Fan, X. Hu, and R. S. Chen, "Multilevel Complex Source Beam Method for Electromagnetic Scattering Problems," IEEE Antennas and Wireless Propagation Letters, vol. 14, pp. 843-846, 2015.

[18] K. C. Wang, Z. H. Fan, M. M. Li, and R. S. Chen, "An effective MoM solution with nested complex source beam method for electromagnetic scattering problems," Institute of Electrical and Electronics Engineers. Transactions on Antennas and Propagation, vol. 64, no. 6, pp. 2546-2551, 2016.

[19] K. Tap, Complex source point beam expansions for some electromagnetic radiation and scattering problems [Ph.D. dissertation], The Ohio State University, 2007.

[20] P. F. Gu, Z. H. Fan, and R. S. Chen, "Complex source beam method for electromagnetic scattering problems of dielectric objects," IEEE Antennas and Wireless Propagation Letters, vol. 15, pp. 597-601, 2016. 


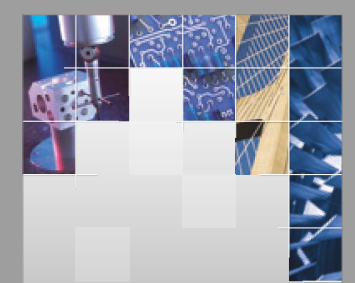

\section{Enfincering}
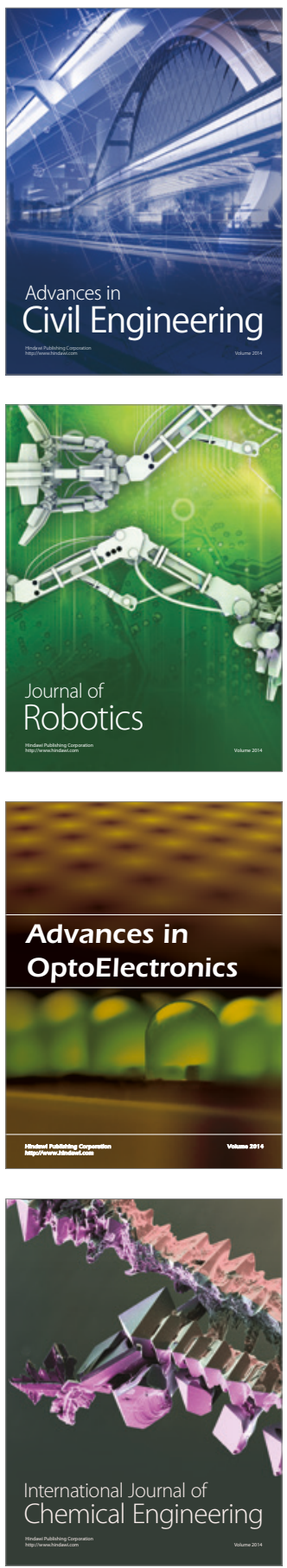

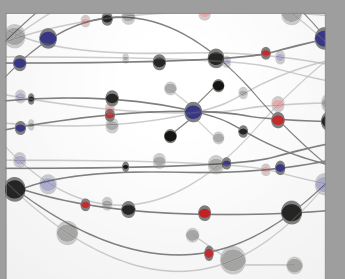

The Scientific World Journal

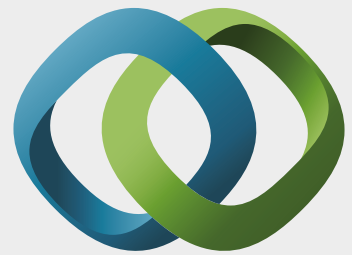

\section{Hindawi}

Submit your manuscripts at

https://www.hindawi.com
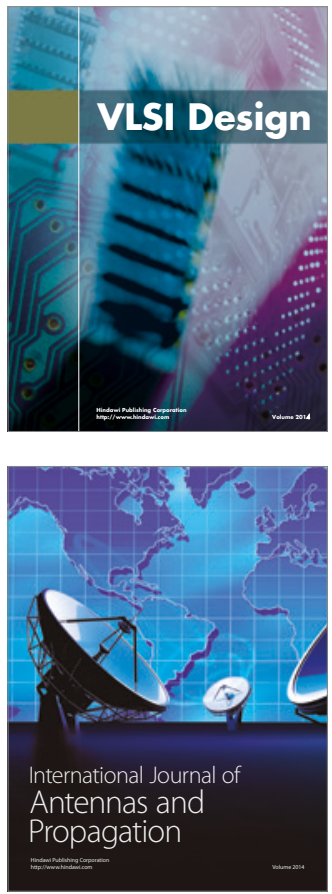

\section{Rotating}

Machinery
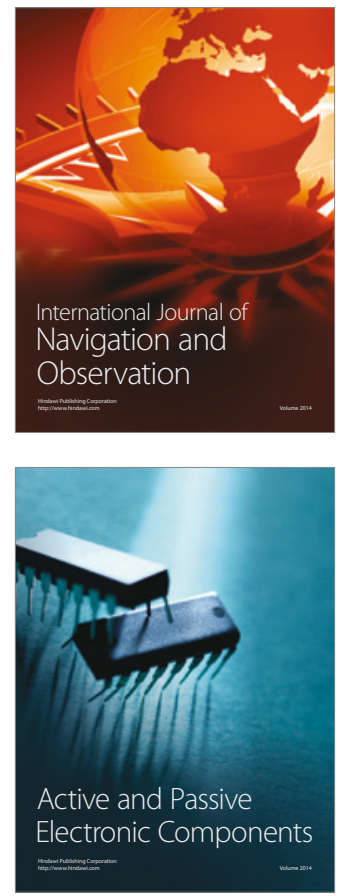
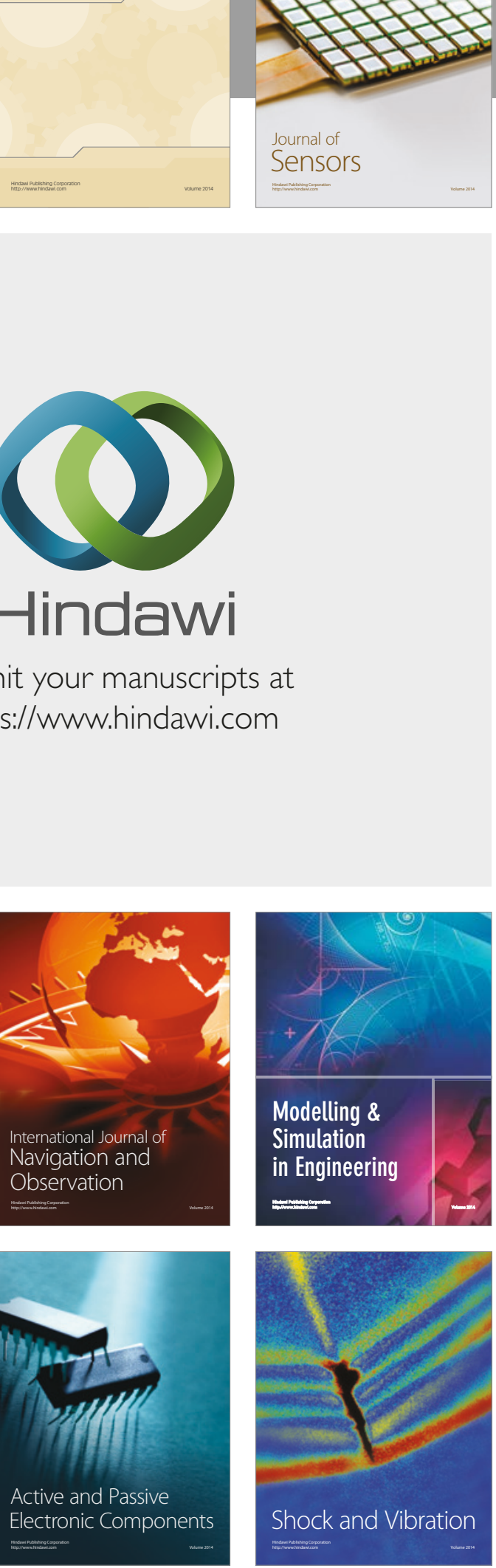
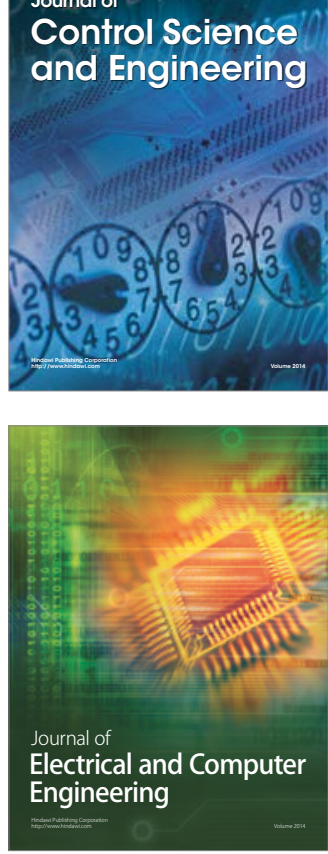

Distributed

Journal of

Control Science

and Engineering
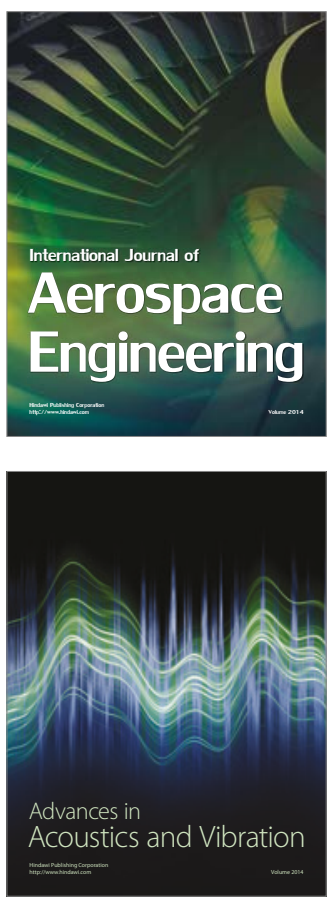

Sensor Networks 